\title{
Nonlinear electrochemical impedance spectroscopy of lithium-ion batteries: Experimental approach, analysis, and initial findings
}

\author{
Matthew D. Murbach, Victor W. Hu, and Daniel T. Schwartz \\ Department of Chemical Engineering \& \\ Clean Energy Institute \\ University of Washington \\ Seattle, WA 98195 \\ dts@uw.edu
}

\begin{abstract}
Nonlinear electrochemical impedance spectroscopy (NLEIS) is a moderateamplitude extension to linear EIS that provides a sensitive and complementary whole-battery diagnostic for charge transfer kinetics, mass transport, and thermodynamics. We present the first full-frequency, second harmonic NLEIS spectra for lithium-ion batteries using commercially available, 1.5 Ah LiNMCIC cells. The mathematical framework for NLEIS shows, and experiments confirm, that moderate-amplitude input modulations can generate a second harmonic output that does not intrinsically corrupt the linear EIS response. Experimental measurements at varied states-of-charge (SoC) and states-of-health $(\mathrm{SoH})$ are used to illustrate and compare NLEIS and EIS data. At low frequencies, the second harmonic NLEIS spectrum is shown to produce a much more distinct response to SoC dependent thermodynamic and diffusion processes than linear EIS. By combining NLEIS and EIS, we are able to characterize degradation in early cell cycling (where cells lost $<1 \%$ of initial capacity). We also show that NLEIS complements the characterization of charge transfer kinetics of linear EIS through the second harmonic sensitivity to symmetry. For example, NLEIS shows that fresh cells have high symmetry charge transfer $\left(\alpha_{a}=\alpha_{c}=0.5\right)$ on both electrodes, whereas early in the cycling there is a shift toward kinetics that favor oxidation on the positive electrode $\left(\alpha_{a, \text { pos }}>0.5, \alpha_{c, p o s}<0.5\right)$. Combined analysis of EIS and NLEIS spectra shows promise for improved parameter estimation and model validation. All experimental data and analysis code for this manuscript can be found on ECSar $X$ iv.
\end{abstract}

\section{Introduction}

The unrelenting drive for higher energy and power dense lithium-ion batteries means the quantity of active materials is increasing at the same time separator thickness and electrolyte volume is decreasing. ${ }^{1}$ Under these conditions, chemical and electrochemical interactions across the whole cell are critical for understanding and optimizing battery performance. Thus, there is a growing need for sensitive and selective in situ diagnostics that support fundamental studies of whole cells. Electrochemical impedance spectroscopy (EIS) is a powerful, noninvasive technique that works by applying

*equal contribution

Preprint. Work in progress. 
a small current or voltage modulation and measuring the linear voltage or current response. ${ }^{2}$ EIS has been used to characterize lithium-ion battery properties such as the solid-electrolyte interphase (SEI) layers, ${ }^{3,4}$ mass transfer and kinetics of the electrode materials, ${ }^{5,6}$ and degradative and capacity loss mechanisms. ${ }^{7,8}$ EIS experiments are traditionally analyzed using equivalent circuit analogs, but linearized physics-based models are also available. ${ }^{9-11}$ While EIS is a widely used and valuable technique, batteries are inherently nonlinear systems, and the linear nature of EIS can lead to degeneracy; that is, a single EIS dataset can often be represented equally well by different linear models ${ }^{12}$ and circuit analogs. ${ }^{13}$

A natural extension to EIS uses moderately larger amplitude perturbations of current or voltage to drive the battery into a weakly nonlinear regime. This variant of EIS, which we call nonlinear EIS (NLEIS), breaks the degeneracy of linearization and contains complementary information to EIS. ${ }^{12}$ In the weakly nonlinear regime, a moderate-amplitude, single-sine input of current or voltage produces one or more measurable (but small) higher harmonics of the fundamental frequency in the output Fourier spectrum. These higher harmonics have often been treated as evidence of a "corrupted" linear response to be eliminated from EIS measurements; ${ }^{14-18}$ however, NLEIS spectra can be used to elucidate information about the nonlinear dynamics of a system that cannot be probed with EIS alone. NLEIS has previously been used in studies of corrosion, ${ }^{19-21}$ fuel cell mechanisms, ${ }^{12,22,23}$ mass transport-controlled systems, ${ }^{24-26}$ chemical sensing, ${ }^{27-29}$ as well as lithium-ion batteries. ${ }^{30,31}$ Lvovich and Smiechowski were able to characterize degradation in industrial lubricants through monotonic trends in the second harmonic NLEIS responses, despite no noticeable trend in the linear responses. ${ }^{32}$ Wilson et al. used physical models to distinguish the rate determining mechanism of the oxygen-reduction reaction on solid oxide fuel cell electrodes, despite the models being degenerate in their linear response. ${ }^{12} \mathrm{Xu}$ and Riley demonstrated that the transfer coefficients, which have typically been experimentally difficult to determine, can be found through the use of even harmonics in the well-characterized ferri-ferrocyanide redox couple. ${ }^{33}$ Heubner et al., further used the same principles to determine the transfer coefficients in lithium iron-phosphate electrodes. ${ }^{34}$ Recently, higher harmonic responses in lithium-ion batteries have been studied through a total-harmonic distortion analysis to examine nonlinear Faradaic processes and battery degradation. ${ }^{30,35-37}$

In these past studies, it is important to make distinctions between nonlinear measurements in the weakly nonlinear regime, where the measurable harmonics are few in number and the voltage modulation is less than the thermal voltage, $\frac{R T}{n F}$ (approximately $25 \mathrm{mV}$ for a single electron transfer at room temperature), ${ }^{12,19-22,24-26,31}$ versus those studies that use much larger modulations of current or voltage and produce many harmonics. ${ }^{30,35-37}$ The NLEIS analysis presented here is only appropriate for the weakly nonlinear response.

NLEIS measurements are an interesting complement to EIS, in part, because the measurements do not necessarily require new equipment from traditional EIS. ${ }^{15,33}$ Here we show how small changes in the experimental procedures and signal processing for EIS can allow researchers to calculate higher harmonic NLEIS spectra. These results are used in conjunction with an extension of the pseudo-two-dimensional (P2D) impedance battery model that includes the nonlinear harmonic response $^{31}$ to gain insight into the physicochemical behavior of commercial Samsung 18650 lithiumion cells. 


\section{Methods}

\subsection{Cycling procedure of the lithium-ion battery}

Cycling measurements on commercially available Samsung 1.5 Ah LiNMCIC cells (INR 18650$15 \mathrm{M})$ were made using a Maccor $4000 \mathrm{M}$ battery cycler in a controlled temperature environment of $25^{\circ} \mathrm{C}$ using a Maccor MTC-020 chamber. In these experiments, all cells were first subjected to five formation cycles using a $\mathrm{C} / 2$ charge and discharge rate within the specified voltage window $(2.5 \mathrm{~V}$ to $4.2 \mathrm{~V}$ ). To study the effects of aging, NLEIS measurements were conducted on new cells and cells that had been cycled for 100 cycles. The full cycles consisted of a 2C (3 A) constant current charge from $2.5 \mathrm{~V}(0 \% \mathrm{SoC})$ to $4.2 \mathrm{~V}(100 \% \mathrm{SoC})$, a constant voltage at $4.2 \mathrm{~V}$ until a $100 \mathrm{~mA}$ current cutoff was reached, and a constant current discharge at $2 \mathrm{C}$.

\subsection{Measuring the nonlinear impedance of a lithium-ion battery}

We demonstrate the full-spectrum application of nonlinear electrochemical impedance spectroscopy for batteries using an Autolab PGSTAT128N potentiostat/galvanostat with the frequency response analyzer module (Autolab FRA32). The measurements were made at a controlled temperature of $25^{\circ} \mathrm{C}$ and used a four-electrode configuration with voltage sensing at the cell terminals.

A single cosine current modulation waveform with frequency $\omega_{1}$ and amplitude $\Delta I$ was sent to the galvanostat

$$
\hat{I}\left(t ; \Delta I, \omega_{1}\right)=\Delta I \operatorname{Re}\left\{\exp \left(j \omega_{1} t\right)\right\},
$$

where $j=\sqrt{-1}$ and Re denotes the real component of the bracketed function. The notation $I\left(t ; \Delta I, \omega_{1}\right)$ can be generalized as $X(a ; b, c, \ldots)$ to show $X$ is the dependent variable, $a$ is an independent variable, and $b, c, \ldots$ are experimental parameters of the solution. The actual time domain current perturbation applied to the battery by the galvanostat, $I\left(t ; \Delta I, \omega_{1}\right)$, and the measured voltage response, $V\left(t ; \Delta I, \omega_{1}\right)$, were recorded using input modulations of $0.3,0.4$, and $0.5 \mathrm{~A}$ over a logarithmically spaced frequency range from $10 \mathrm{kHz}$ to $3.16 \mathrm{mHz}$. Time domain data were recorded after the system had reached a steady-periodic state. A minimum of 10 steady-periodic cycles were logged with $N=4096$ synchronous time domain measurements for both current and voltage. A rest time of 30 minutes was provided between each frequency sweep to provide a consistent baseline.

Measurements at specific states-of-charge were carried out by adding a specified amount of charge to a fully discharged battery (discharged to $2.5 \mathrm{~V}$ and held until the current reached $1 \mathrm{~mA}$ ). The amount of charge to add was determined by capacity measurements at $\mathrm{C} / 10$ cycling. The voltages of specific states-of-charge were recorded after a four-hour rest to allow the cell to equilibrate. ${ }^{38}$ For subsequent NLEIS experiments, each cell was charged to the desired voltage designated by the baseline (summarized in Table A1) through a $\mathrm{C} / 10$ rate, and then subjected to a two-hour constant voltage charge at the desired voltage. The cells were then allowed to equilibrate for an additional two hours at open circuit.

Fast Fourier transformation (FFT) of a steady-periodic input current of the form given by Equation 1, theoretically results in a very simple spectrum,

$$
\operatorname{FFT}\left\{I\left(t ; \Delta I, \omega_{1}\right)\right\}=\Delta I,
$$

at the frequency $\omega_{1}$, and zero at all other frequencies. The resulting voltage spectrum, however, often contained complex Fourier coefficients at integer multiples, $k$, of the fundamental frequency, 
$\omega_{1}$,

$$
\operatorname{FFT}\left\{V\left(t ; \Delta I, \omega_{1}\right)\right\}=\tilde{V}\left(k \omega_{1} ; \Delta I\right) .
$$

The experimental reality of our galvanostat was that harmonic distortions of the pure cosine current were introduced into the ideal spectrum given by Equation 2 when operated at higher frequencies and amplitudes, leading to an input current spectrum of the form:

$$
\operatorname{FFT}\left\{I\left(t ; \Delta I, \omega_{1}\right)\right\}=\tilde{I}^{\prime}\left(1 \omega_{1} ; \Delta I\right)+T H D
$$

where $T H D$ is the total harmonic distortion of the single sine input signal and $\tilde{I}^{\prime}\left(1 \omega_{1} ; \Delta I\right)$ is the real Fourier coefficient at the fundamental frequency of the current perturbation (closely approximating $\Delta I)$. To ensure nonlinear experimental results presented here are from nonlinearity in the battery, not the power amplifier, we only report second harmonic data up to a frequency of $10 \mathrm{~Hz}$ for this instrument, where THD in the input was always below 1.0\%. Linear EIS is reported for the entire frequency range.

The complex Fourier voltage spectrum had a pure harmonic structure comprised of signals at integer $k$ values of the fundamental frequency $\omega_{1}$. The lack of sub-harmonic $(0<k<1)$ or anharmonic (non-integer $k$ ) signals in the output voltage spectrum indicated that the batteries (and measurement instrument) were stable, in all cases, to the finite amplitude current modulations. However, noise and imperfect sampling did lead to a small, continuous baseline in the spectrum. The baselines for the linear $(k=1)$ and second harmonic $(k=2)$ peaks were subtracted using a quadratic polynomial fit to several anharmonic baseline points adjacent to each harmonic peak of interest.

The amplitude of each voltage harmonic depends on the current modulation amplitude, $\Delta I$. Prior research has shown that the weakly nonlinear response of the battery can be expressed as a power series in perturbation amplitude such that, ${ }^{12,25,26}$

$$
\tilde{V}\left(k \omega_{1} ; \Delta I\right)=\sum_{r=0}^{\infty} \Delta I^{k+2 r} \tilde{Z}_{k, k+2 r}\left(\omega_{1}\right)
$$

where the resulting double-indexed coefficients, $\tilde{Z}_{k, p}\left(\omega_{1}\right)$, are pure frequency dispersion coefficients - we will call them linear and nonlinear impedance coefficients - corresponding to harmonic number, $k$, and order, $p$. The double-indexed coefficient $\tilde{Z}_{1,1}\left(\omega_{1}\right)$ is identically equal to the normal (linear) electrochemical impedance of the system. $\tilde{Z}_{2,2}\left(\omega_{1}\right)$ is the leading order nonlinear electrochemical impedance derived from the second harmonic of the voltage response to a pure cosine input current. The impedance coefficients for $p>k$, represent higher order corrections to the leading order $k=p$ solutions. In this work, we present an experimental and computational evaluation of the leading order terms in Equation 5,

$$
\begin{gathered}
\tilde{V}\left(1 \omega_{1} ; \Delta I\right)=\Delta I \tilde{Z}_{1,1}\left(\omega_{1}\right) \\
\tilde{V}\left(2 \omega_{1} ; \Delta I\right)=\Delta I^{2} \tilde{Z}_{2,2}\left(\omega_{1}\right) .
\end{gathered}
$$

From the complete form of Equation 5, it is possible to estimate how error scales when we truncate the series and use Equation 6 to determine the traditional linear electrochemical impedance, and Equation 7 to find the second harmonic NLEIS spectrum. Specifically, the error associated with using Equation 6 scales as $\sim O\left(\Delta I^{3}\right)$ and the error from using Equation 7 scales as $\sim O\left(\Delta I^{4}\right)$.

All of the data presented in this paper, including additional experiments showing reproducibility across several identical Samsung cells and data for analyzing the frequency-dependent galvano- 

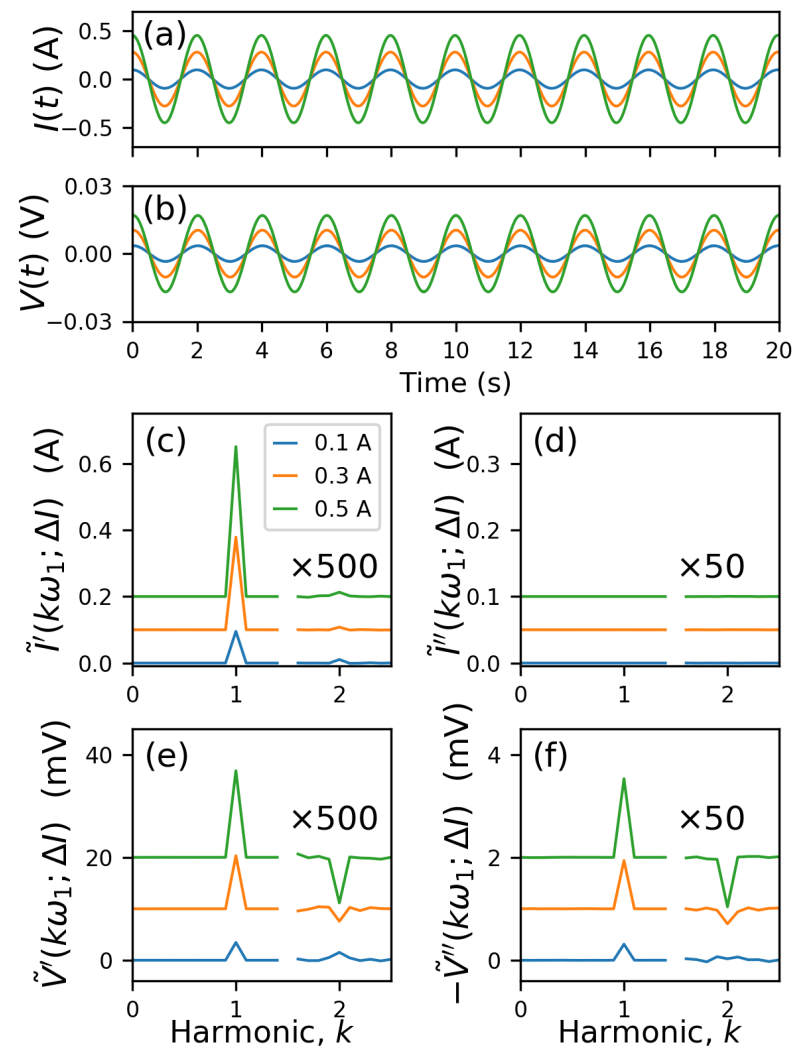

Figure 1: Measured time domain current (a) and voltage (b) response for a commercial 1.5 Ah LiNMCIC cell excited by a $\omega_{1}=0.5 \mathrm{~Hz}$ current perturbation of varying amplitudes $(\Delta I)$ ranging from $0.1 \mathrm{~A}$ to $0.5 \mathrm{~A}$. These signals are transformed into the frequency domain to enable analysis of the real (c, e) and imaginary (d, f) components at different harmonics, $k$. Offsets are added for clarity and the real and imaginary values for $k>1.5$ are multiplied by $500 \times$ and $50 \times$, respectively, to be on the same scale. Voltage signal has mean open circuit value of $3.643 \mathrm{~V}$ substracted.

stat harmonic distortion, along with all of the software used for signal processing and analysis, can be found in the Supplementary Jupyter Notebook. ${ }^{39}$

\section{Results}

\subsection{Current perturbation and voltage response signals}

Typical time domain measurements of the input current and output voltage response from a Samsung high power LiNMClC cell are shown for a frequency of $\omega_{1}=0.5 \mathrm{~Hz}$ in Figure 1a and 1b, respectively, for three different current modulation amplitudes $(\Delta I=0.1 \mathrm{~A}, 0.3 \mathrm{~A}$, and $0.5 \mathrm{~A})$. Time domain data of this nature, measured at different states-of-charge, frequencies, and amplitudes, are the raw data for our method.

In the time domain, the current (Figure 1a) appears to be a pure cosine. The output voltage response (Figure 1b) also appears to be a sinusoidal signal, with a phase and amplitude that depends on the input frequency and amplitude of the modulated current. For these high-power $1500 \mathrm{mAh}$ cells, a current modulation of $100 \mathrm{~mA}$ is seen to produce a voltage modulation of about $4 \mathrm{mV}$ around the open circuit voltage, whereas the largest current modulation of $500 \mathrm{~mA}$ produces roughly 20 
$\mathrm{mV}$ of voltage modulation around the open circuit voltage. In EIS, a $4 \mathrm{mV}$ modulation would be expected to fall in the linear response regime of nearly any electrochemical system. In contrast, a 20 $\mathrm{mV}$ modulation would generally be considered large enough to drive a weakly nonlinear response. For all of the NLEIS experiments reported here, we use current modulations between 300 and 500 $\mathrm{mA}$; for the Samsung cells under test, the modulated voltages are in the 10s of millivolts, or weakly nonlinear. We also do select experiments with $33 \mathrm{~mA}$ current modulation to determine impedances under traditional, very small amplitude linear conditions.

Details of the phase, amplitude, and harmonic content of the input current and output voltage signals are more evident in frequency domain spectra determined through FFT of the time domain signals, as shown in Figures 1c-1f. The real and imaginary current spectra (Figure 1c and 1d, respectively) show that the input is a purely real signal. A tiny amplifier-induced second harmonic signal $(k=2)$ is made evident by multiplying that portion of the spectrum by $500 \times$, demonstrating that the actual galvanostat amplifier has a spectrum represented by Equation 4, but it is close to the ideal input given by Equation 2. In all that follows, we use the measured value $\tilde{I}^{\prime}\left(1 \omega_{1} ; \Delta I\right)$ rather than the ideal value $\Delta I$, when computing experimental impedances via Equations 6 and 7 . As noted, the difference between the measured $\tilde{I}^{\prime}\left(1 \omega_{1} ; \Delta I\right)$ and $\Delta I$ is generally small.

The real and imaginary frequency domain voltage spectra (Figures 1e and 1f, respectively) show several features not easily seen in time domain measurements. In the frequency domain, the phase shift and magnitude of each harmonic in the voltage spectra can be quantified based by the magnitudes of the real and imaginary components. Qualitatively, one can see that the first harmonic $(k=1)$ in voltage grows linearly with $\tilde{I}^{\prime}\left(1 \omega_{1} ; \Delta I\right)$ values from Figure 1c, as predicted by Equation 6. Likewise, the second harmonic $(k=2)$ in voltage appears to grow at a super-linear rate with $\tilde{I}^{\prime}\left(1 \omega_{1} ; \Delta I\right)$; Equation 7 suggests a quadratic dependence. The signs and ratios of the real and imaginary components for the $k=2$ peaks are different than the $k=1$ peaks at the fundamental frequency.

\subsection{Amplitude dependence of voltage harmonics}

To calculate the linear (traditional EIS) and nonlinear second harmonic impedance coefficients for the data in Figure 1, at frequency $\omega_{1}=0.5 \mathrm{~Hz}$, we use the real and imaginary $k=1$ and $k=2$ data along with Equations 6 and 7. Figure 2 shows the amplitude dependence of the voltage harmonics in Figures 1e and 1f, as a function of $\tilde{I}^{\prime}\left(1 \omega_{1} ; \Delta I\right)$, the $k=1$ peak in Figure 1c. The impedance coefficients $\tilde{Z}_{1,1}\left(\omega_{1}\right)$ and $\tilde{Z}_{2,2}\left(\omega_{1}\right)$ are the best-fit coefficients from the polynomials in Equations 6 and 7 , where $\tilde{I}^{\prime}\left(1 \omega_{1} ; \Delta I\right)$ rather than $\Delta I$ is used. To retain the phase information in the doubleindexed impedance coefficients, the real (Figure 2a) and imaginary (Figure 2b) components of the harmonic response are fit separately.

The solid lines in Figure 2 are the best-fit curves, and the dashed regions represent the extrapolations outside the three fit data points. The $k=1$ line shows an independent, very small amplitude data point at $0.033 \mathrm{~A}$, representing a measurement taken under conditions well away from any experimentally detectable voltage second harmonic. The $0.033 \mathrm{~A}$ points are seen to lie on the extrapolated linear fit made from the $0.3,0.4$, and $0.5 \mathrm{~A}$ data. The calculated $\tilde{Z}_{1,1}$ value from fitting the three larger amplitude points in Figure 2a is $\tilde{Z}_{1,1}=37.31 \pm 0.17 \mathrm{~m} \Omega$. The value measured from the traditional small amplitude 0.033 A modulation, where there is no $k=2$ peak, is $\tilde{Z}_{1,1}=37.37 \mathrm{~m} \Omega$, well within the confidence interval of the larger amplitude value. Moreover, all $\tilde{Z}_{1,1}\left(\omega_{1}\right)$ linear impedances calculated from the $0.3,0.4$, and 0.5 A points were found to satisfy Kramers-Kronig relations ${ }^{40}$ to within $1.0 \%$ (see the Supplementary Jupyter Notebook). ${ }^{39}$ 

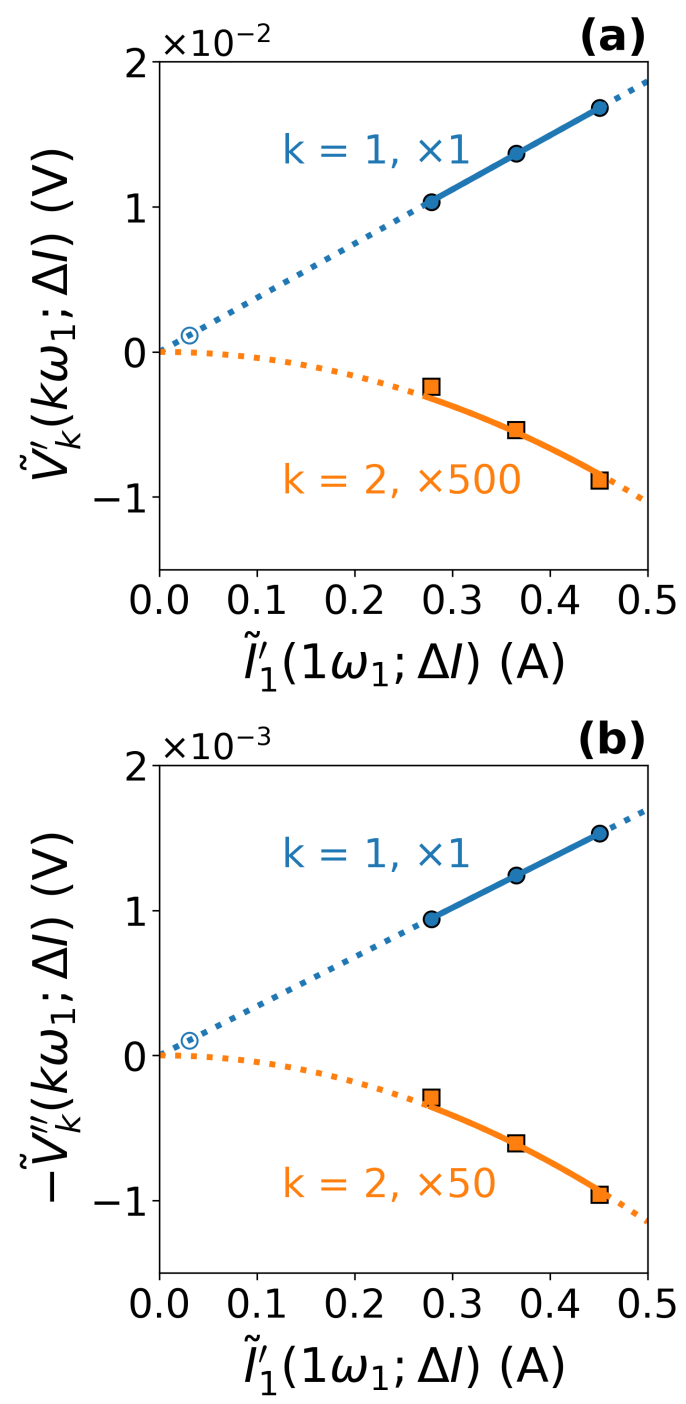

Figure 2: Amplitude dependence of the real (a) and imaginary (b) components of the Fourier transformed voltage response $\left(\omega_{1}=0.5 \mathrm{~Hz}\right)$ as a function of the current perturbation amplitude, $\Delta I$. The first $(k=1,0)$ and second $(\mathrm{k}=2, \square)$ voltage harmonics are fit to linear, $\tilde{I}^{\prime}\left(1 \omega_{1} ; \Delta I\right) \tilde{Z}_{1,1}\left(\omega_{1}\right)$, and quadratic, $\tilde{I}^{\prime}\left(1 \omega_{1} ; \Delta I\right)^{2} \tilde{Z}_{2,2}\left(\omega_{1}\right)$, functions of the measured perturbation amplitude, respectively. The real and imaginary second harmonic data are multiplied by 500 and 50, respectively, to place them on the same scale as the linear response. The data shown are for cell 5 at $30 \%$ SoC. 
The results shown in Figure 2 are consistent across our wide range of frequency response data, and they support the hierarchical mathematical structure of Equation 5. Specifically, our results and the math show it is possible to simultaneously, self-consistently, and accurately determine the $\tilde{Z}_{1,1}$ spectrum from $k=1$ voltage data (that scale with $\Delta I$ ) and the $\tilde{Z}_{2,2}$ spectrum from $k=2$ voltage data (that scales with $\Delta I^{2}$ ), because the leading order truncation error in $\tilde{Z}_{1,1}$ scales with $\sim O\left(\Delta I^{3}\right)$. Stated another way, there is no fundamental reason the presence of an easily detected second harmonic in the voltage spectrum is indicative that the linear impedance is corrupted by nonlinear effects. Instead, the math suggests that a cautionary signature of nonlinearity corrupting EIS is the presence of a third harmonic in the voltage spectrum (i.e., $k=3$ signature), because that harmonic scales with $\Delta I^{3}$, just like the truncation error in $\tilde{Z}_{1,1}$. The experimental implication is that we chose current modulation amplitudes of $0.3,0.4$, and $0.5 \mathrm{~A}$ for the battery under test, because these values are a good compromise between measurable second harmonics but negligible third harmonics in the voltage spectra.

As noted in the discussion of Equation 4, the galvanostat (like all amplifiers) is imperfect and generates harmonic distortions; these instrument-induced signals are expected to appear in the input current and output voltage spectra. To assess the magnitude and remove galvanostat-generated second harmonic in the voltage output, we use a $\tilde{Z}_{1,1}^{\prime}=21 \mathrm{~m} \Omega$ resistive wire that is comparable in magnitude to the impedance of the batteries under test, but has no intrinsic nonlinear harmonic generating behavior. Using the same analysis of $k=2$ voltage spectra as shown in Figure 2, we find the purely linear resistor produced a frequency invariant $\tilde{Z}_{2,2}^{\prime i n}=4.56 \times 10^{-5} \frac{\Omega}{A}$ that can be entirely associated with the instrument. In all of the second harmonic NLEIS spectra we report, this instrument value is subtracted from each $\tilde{Z}_{2,2}\left(\omega_{1}\right)$ point to remove the small instrument offset.

\subsection{Nyquist representation of linear and nonlinear EIS spectra}

Measuring the linear and nonlinear impedance coefficients over a range of frequencies provides insights into the system at different physicochemical timescales. Figure 3 shows a Nyquist representation of the experimentally measured linear EIS spectrum, $\tilde{Z}_{1,1}\left(\omega_{1}\right)$, and second harmonic NLEIS spectrum, $\tilde{Z}_{2,2}\left(\omega_{1}\right)$, for a fresh cell at $50 \%$ state-of-charge (SoC). The linear response shown in Figure $3 \mathrm{a}$ is a typical EIS spectrum for a lithium-ion battery. Figure $3 \mathrm{a}$ has a high frequency realaxis intercept $(>1 \mathrm{kHz})$ related to the internal resistance of the battery, a mid-range frequency arc ( $1 \mathrm{kHz}$ to $1 \mathrm{~Hz}$ ) attributable to the coupled charge transfer kinetics and double-layer capacitance of the porous electrodes, and a low frequency tail $(<1 \mathrm{~Hz})$ attributable to diffusive transport and thermodynamics. The linear EIS spectra we are measuring can be thought of as the sum of two half-cell impedances for the positive and negative electrodes,

$$
\tilde{Z}_{1,1}=\tilde{Z}_{1,1}^{+}+\tilde{Z}_{1,1}^{-}
$$

where each half cell is defined at the mid-plane of the separator. Analysis of linear EIS spectra using equivalent circuit modeling is common in the literature, where circuit elements can be related to characteristics of the electrodes and separator. ${ }^{2,9,41,42}$

Prior work ${ }^{31}$ shows that the second harmonic response is highly sensitive to several forms of symmetry in the system under test. The second harmonic NLEIS spectra we are measuring can be thought of as the difference between the half-cell impedances for the positive and negative electrodes,

$$
\tilde{Z}_{2,2}=\tilde{Z}_{2,2}^{+}-\tilde{Z}_{2,2}^{-}
$$



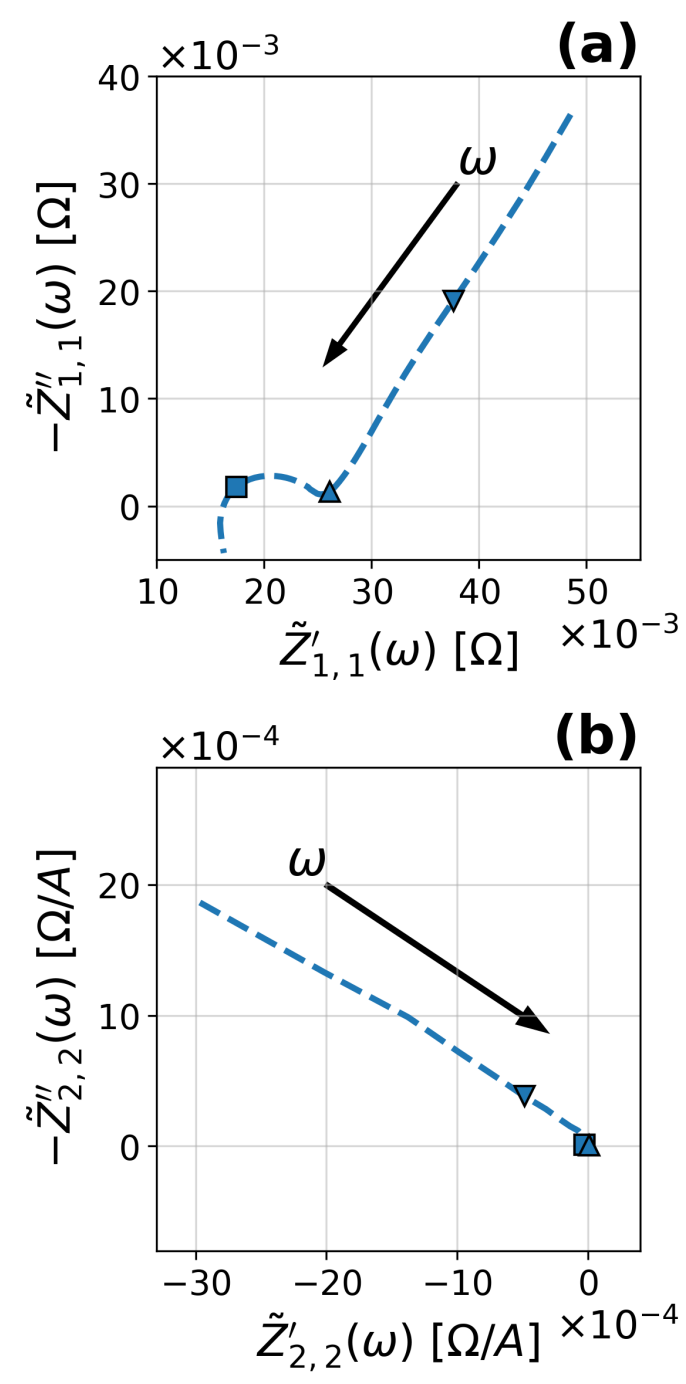

Figure 3: Nyquist plots of measured first (a) and second (b) harmonic coefficients, $\tilde{Z}_{1,1}\left(\omega_{1}\right)$ and $\tilde{Z}_{2,2}\left(\omega_{1}\right)$ of "fresh" battery at $50 \%$ SoC. The data at $1 \mathrm{kHz}(\square), 1 \mathrm{~Hz}(\mathbf{\Delta})$, and $10 \mathrm{mHz}(\nabla)$ are labeled for clarity.

In a symmetric cell, where the positive and negative electrodes are identical, $\tilde{Z}_{2,2}$ will be identically zero for all frequencies. Of course, a functional battery is not a symmetric cell because thermodynamic differences in each electrode material largely defines the battery. Figure $3 b$ shows that essentially all of the $\tilde{Z}_{2,2}$ response, for a fresh cell at $50 \%$ SoC, occurs at frequencies below $1 \mathrm{~Hz}$, where cell thermodynamics and diffusive transport are expected to be the dominating physics.

In the mid-frequency regime $(>1 \mathrm{~Hz})$, where interfacial kinetic/capacitive processes dominate the linear EIS spectrum of Figure 3 a, we see negligible $\tilde{Z}_{2,2}$ second harmonic response. In our fullphysics model of lithium ion batteries, the lack of kinetic/capacitive response in $\tilde{Z}_{2,2}$ was attributed to high symmetry charge transfer where $\alpha_{a}=\alpha_{c}$ in the Butler-Volmer expression. ${ }^{31}$ To get a basic understanding of charge transfer symmetry in the linear and nonlinear impedance, we carry out a 
Taylor series expansion of the Butler-Volmer equation,

$$
j=i_{0}\left[\exp \left(\frac{\alpha_{a} F}{R T} \eta\right)-\exp \left(\frac{-\alpha_{c} F}{R T} \eta\right)\right]
$$

and include the first two terms

$$
j=\left[\frac{i_{0}\left(\alpha_{a}+\alpha_{c}\right) F}{R T}\right] \eta+\left[\frac{i_{0}\left(\alpha_{a}^{2}-\alpha_{c}^{2}\right) F^{2}}{2 R^{2} T^{2}}\right] \eta^{2}+O\left(\eta^{3}\right)
$$

where $i_{0}$ is the exchange current density, $\alpha_{a}$ and $\alpha_{c}$ are the anodic and cathodic transfer coefficients, and $F, R$, and $T$ are Faradays constant, the gas constant, and the temperature, respectively. The first term on the right-hand side of Equation 11 is the inverse of the classic linear charge transfer resistance whereas the second term describes the leading order nonlinear behavior. Note that the second term is zero when charge transfer is symmetric with $\alpha_{a}=\alpha_{c}$, but can be either positive or negative when $\alpha_{a} \neq \alpha_{c}$.

The classic, linear charge transfer resistance in EIS is insensitive to symmetry because $\alpha_{a}+\alpha_{c}$ is a constant (the number of electrons transferred) for each electrode. On the other hand, the second term of Equation 11, when combined with Equation 9, provides a symmetry-dependence that is consistent with our full-physics battery model behavior when $\alpha_{a}=\alpha_{c}, \alpha_{a}>\alpha_{c}$ or $\alpha_{a}<\alpha_{c}$ for either or both electrodes. ${ }^{31}$ Thus, we can think of the second term in Equation 11 as being related to a "second harmonic charge transfer resistance" for each electrode. The absence of second harmonic NLEIS features at interfacial kinetic/capacitive frequencies in Figure 3b suggests charge transfer on both fresh electrodes is symmetric.

\subsection{State-of-Charge (SoC) dependence for fresh cells}

The full physics theory for linear EIS and second harmonic NLEIS includes explicit state-of-charge (SoC) dependence through derivatives of the open circuit potential of each electrode, and implicit dependence on parameters such as diffusivities, interfacial capacitances, and charge transfer parameters. ${ }^{31}$ Specifically, linear EIS response depends on the first derivatives of the open circuit voltages with respect to intercalated lithium content; the first derivative is always negative for either electrode, but the magnitudes can vary widely by material and SoC. The second harmonic NLEIS response depends on both the first and second derivative with respect to intercalated lithium content; the second derivative can be positive, negative, or zero for either electrode. As a result of the additional parameters, their varied signs, and the fact the response comes from differences between electrodes, per Equation 9, our theory suggests that the thermodynamics/diffusion dominated regions of second harmonic NLEIS spectra can display a much richer set of SoC-dependent behaviors compared to the Warburg-like behavior expected for linear EIS. ${ }^{31}$

Figure 4 shows the linear EIS and second harmonic NLEIS spectra as the $\mathrm{SoC}$ is varied from $50 \%$ (same as Figure 3) to 10\% in a fresh cell. The accompanying Supplemental Jupyter Notebook includes data for other SoCs, but they are excluded from Figure 4 for clarity. ${ }^{39}$ The series of linear EIS spectra in Figure 4a all have characteristic mid-frequency arcs associated with interfacial kinetic/capacitive behavior $\left(\omega_{1}>1 \mathrm{~Hz}\right)$ and low frequency thermodynamic/diffusive tails $\left(\omega_{1}<1 \mathrm{~Hz}\right)$. The interfacial charge transfer impedance grows systematically as the SoC is reduced. The low frequency EIS tail displays subtle changes in length with state-of-charge, being longest at 50\% SoC, shortest at $30 \%$, and longer again at $10 \%$. Based on the full physics EIS model presented elsewhere, the mid-frequency changes are likely to be driven by SoC-dependent changes in exchange current density $\left(i_{0}\right)$ and double-layer capacitance $\left(C_{d l}\right)$ on one or both electrodes. The low frequency re- 

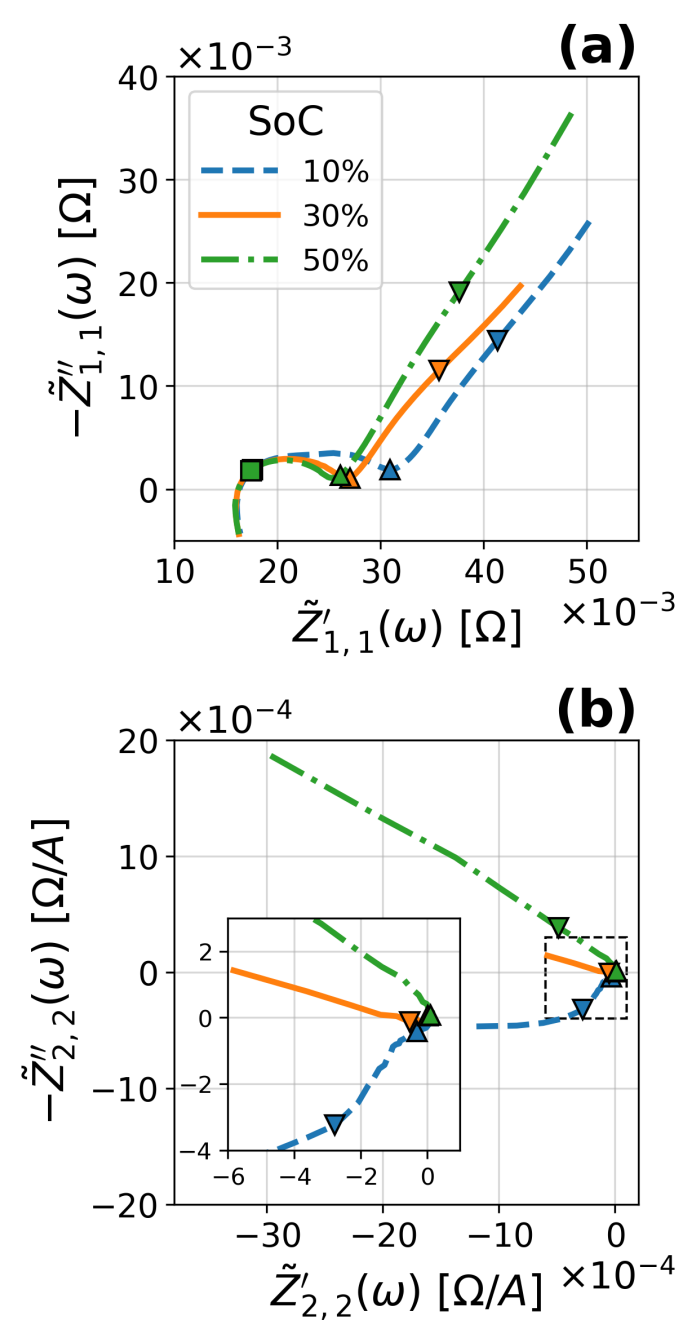

Figure 4: Nyquist plots of measured first (a) and second (b) harmonic coefficients, $\tilde{Z}_{1,1}\left(\omega_{1}\right)$ and $\tilde{Z}_{2,2}\left(\omega_{1}\right)$, for a "fresh" cell at several states-of-charge. The data at $1 \mathrm{~Hz}(\boldsymbol{\Lambda})$ and $10 \mathrm{mHz}(\boldsymbol{\nabla})$ are labeled for clarity and the inset in (b) shows the region near the origin in more detail.

sponse is typically driven by SoC-dependent changes in the first derivative of open circuit voltage and solid-state diffusivity on one or both electrodes, though other transport processes can also come into play. ${ }^{11,31}$ The effects of these changes are additive, per Equation 8.

The second harmonic spectra in Figures $4 \mathrm{~b}$ show much greater variation in behavior than the linear EIS as the SoC changes. Despite large variation in the low-frequency region, no spectra show significant responses in the interfacial kinetic/capacitive regime $\left(\omega_{1}>1 \mathrm{~Hz}\right)$, see inset in Figure $4 \mathrm{~b}$. As discussed above, the lack of significant second harmonic response in the mid-frequency regime indicates symmetry of charge transfer over all states-of-charge tested. The complexity of the low-frequency second harmonic NLEIS response is not surprising given the increased number of parameters controlling the behavior, as described above. Moreover, the fact that the NLEIS spectrum results from differences in each electrode, Equation 9, means that subtle changes in one electrode can be amplified. In theory, this increased sensitivity to parameter changes improves parameter identifiability, but the more complex dependencies of NLEIS means that more sophisticated analysis is 
required. We will explore detailed SoC-dependent analysis and parameter estimation of NLEIS spectra in future work.

\subsection{State-of-Health (SoH)}
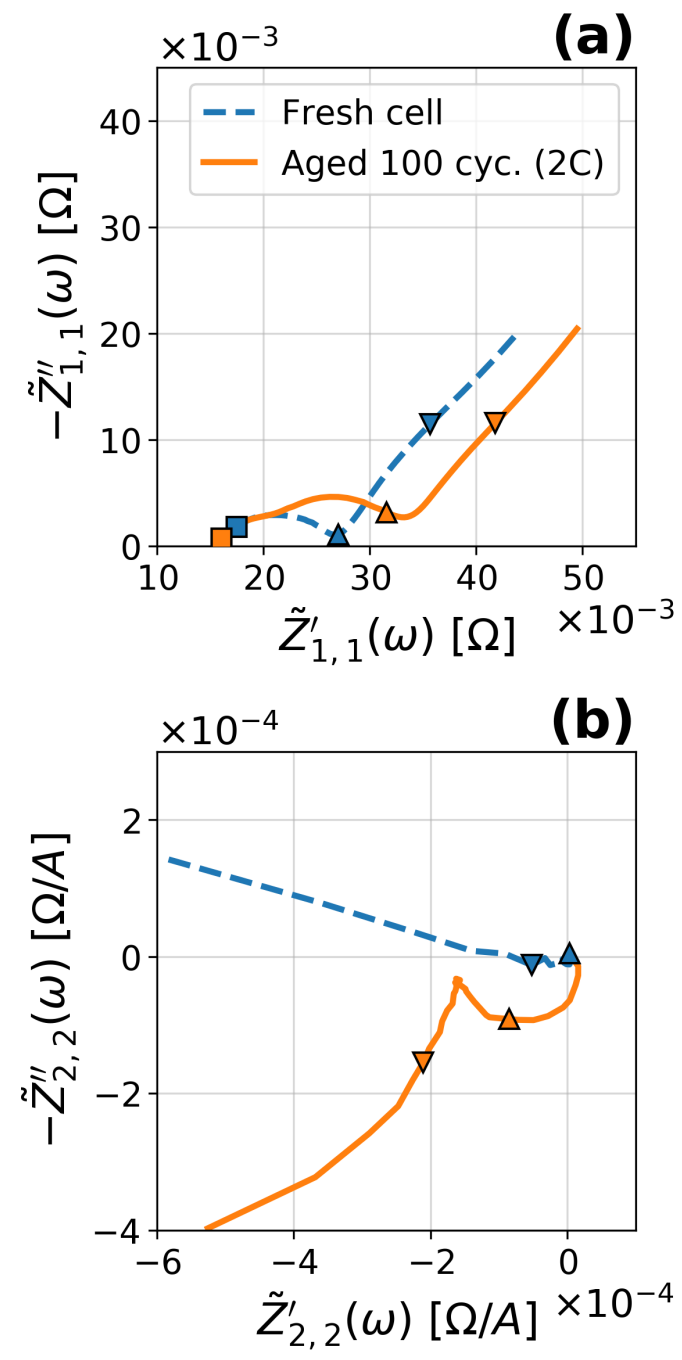

Figure 5: Nyquist plots of measured first (a) and second (b) harmonic coefficients, $\tilde{Z}_{1,1}\left(\omega_{1}\right)$ and $\tilde{Z}_{2,2}\left(\omega_{1}\right)$ for a fresh cell and a cell which has been cycled 100 times at $2 \mathrm{C}$. The data at $1 \mathrm{kHz}(\boldsymbol{\square})$, $1 \mathrm{~Hz}(\boldsymbol{\Lambda})$, and $10 \mathrm{mHz}(\mathbf{\nabla})$ are labeled for clarity. The second harmonic data is truncated at $10 \mathrm{~Hz}$. Cells are at $30 \%$ SoC.

We have begun to explore the sensitivity of second harmonic NLEIS as a method for state-ofhealth $(\mathrm{SoH})$ assessments. Figure 5 shows the linear EIS and second harmonic NLEIS spectra at $30 \%$ SoC for the fresh Samsung power cells and cells that have been charged and discharged at $2 \mathrm{C}$ for 100 cycles. The measured capacity loss from this cycle-dependent aging is small, only $0.8 \%$, implying small changes to physicochemical processes in the battery.

EIS is known to be valuable for prognostic and diagnostic analysis of battery aging. ${ }^{43}$ Here we see the linear EIS response in Figure 5a is noticeably changed in the mid-frequency kinetic/capacitive regime, despite less than $1 \%$ degradation of capacity. In particular, the mid- 
frequency impedance has grown, and two distinct arcs are now visible in the aged cell. For LiNMCIC cells, the growth of a second mid-frequency kinetic/capacitive arc from cycling is typically attributed to an increase in the positive NMC electrode charge transfer resistance. ${ }^{8}$ The highest frequency arcs for fresh and aged cells continue to overlap in Figure 5a, suggesting negligible changes to the negative electrodes from 100 cycle aging. Likewise, aging related changes in the low frequency tail are extremely subtle.

While linear EIS provides useful insights, Figure 5b shows second harmonic NLEIS produces much greater changes from 100 cycle aging than linear EIS; we find all measurable frequencies are distinctly altered in the aged cell. In particular, the emergence of a mid-frequency response in the aged cell indicates a breaking of charge-transfer symmetry for at least one electrode. Combining the EIS interpretation with the new NLEIS results here suggests the positive NMC electrode ages with an increase in linear charge transfer resistance (i.e., decreasing $i_{0}$ ) and that change is accompanied by a breaking of charge transfer symmetry (i.e., $\alpha_{a} \neq \alpha_{c}$ ).

Previous full-physics theory results show that the "second harmonic charge transfer resistance," as we called it above, can be either positive or negative depending on the direction of charge transfer asymmetry and the electrode where that asymmetry arises. ${ }^{31}$ Using these prior results, and attributing all the degradation to the positive electrode, means the charge transfer arc in Figure 5b arises from the anodic transfer coefficient, $\alpha_{a, p o s}$, being larger than the cathodic transfer coefficient, $\alpha_{c, p o s}$. For a positive electrode with $\alpha_{a, p o s}>\alpha_{c, p o s}$, oxidation (charging) is relatively easier (requires lower overpotential) than the equivalent reduction (discharging). This new insight into mechanistic changes in charge transfer cannot be determined from EIS alone, as linearization of the governing equations (and the governing physics) makes EIS wholly insensitive to charge transfer symmetry in either electrode.

As described in Figure 4b, the low frequency tail is sensitive to subtle changes in several thermodynamic and diffusive transport parameters for either or both electrodes. As noted above, the sensitive, multi-parameter nature of the NLEIS low frequency domain makes attention to modeling more essential for interpretation. Overall, second harmonic NLEIS appears to complement linear EIS as a method for probing degradation processes at the earliest stages of cycling.

\subsection{Physics-based modeling of the EIS and NLEIS spectra}

All of the experimental observations we have made in Figures 3-5 can be represented by our previously developed pseudo 2-dimensional (P2D) physics-based model for the linear EIS and second harmonic NLEIS response of a lithium-ion battery. ${ }^{10}$ We are currently building up the computational tools needed for multi-parameter estimation of linear EIS and second harmonic NLEIS based on full-physics parameter estimation fit to experiments. ${ }^{44}$ However, even without best-fit parameters, it is possible to qualitatively demonstrate that important characteristics of the linear and nonlinear response for the battery can be captured by the model. For example, Figure 6 shows simulated spectra that qualitatively mirror the EIS and NLEIS aging results presented in Figure 5; the two sets of model parameters are given in Table A.2.

In the aged cell experiments, the appearance of two distinct kinetic/capacitive arcs in the EIS spectrum was attributable to growth of the charge transfer resistance and an increase in the characteristic "RC" time constants for the positive electrode. The main way the model captures this aging affect is by a large reduction in positive electrode $i_{0}$ and large increase in double-layer capacitances $C_{d l}$ for both electrodes. Changes to the charge transfer symmetry have no effect on the linear EIS. Further, looking at Table A.2, we see that many of the thermodynamic and diffusion 

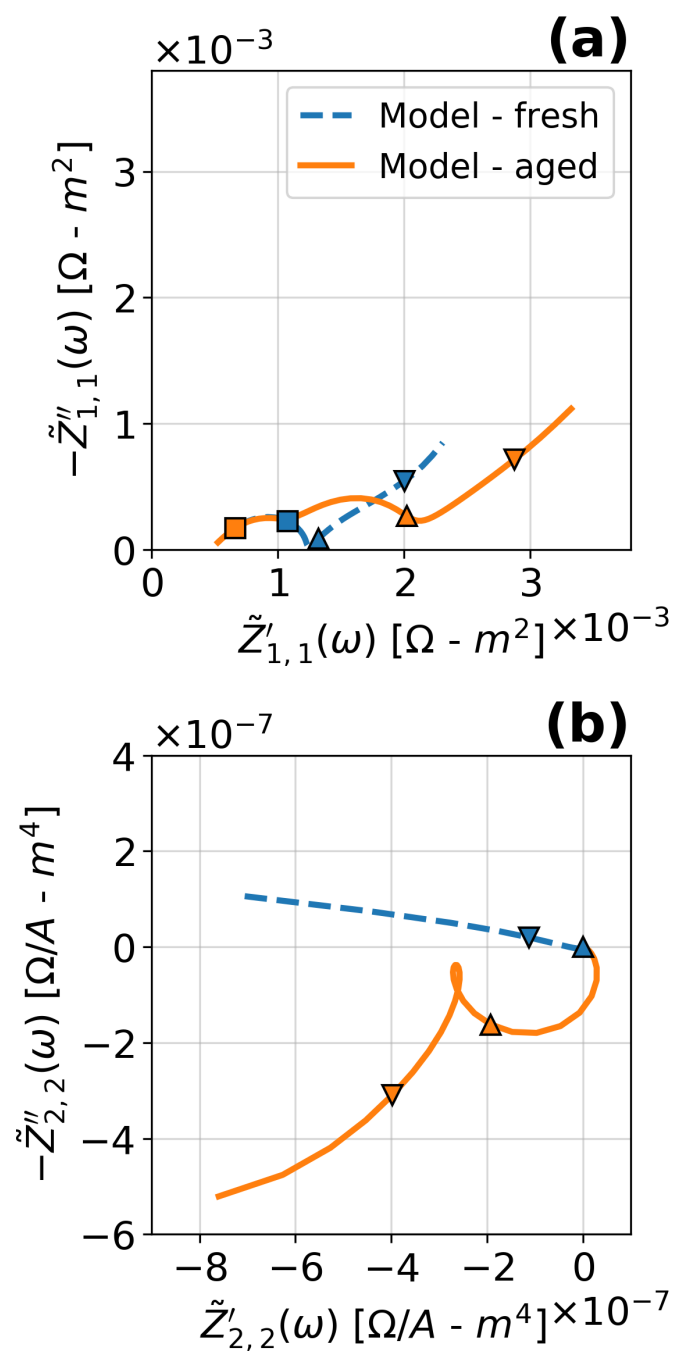

Figure 6: Nyquist plots of computed first (a) and second (b) harmonic coefficients, $\tilde{Z}_{1,1}\left(\omega_{1}\right)$ and $\tilde{Z}_{2,2}\left(\omega_{1}\right)$ from $3.16 \mathrm{mHz}$ to $10 \mathrm{kHz}$. The data at $1 \mathrm{kHz}(\boldsymbol{\nabla}), 1 \mathrm{~Hz}(\boldsymbol{\Lambda})$, and $10 \mathrm{mHz}(\boldsymbol{\nabla})$ are labeled for clarity.

parameters are changed for the aged cell, but these changes only produce subtle differences in the linear EIS spectrum at low frequencies, similar to the experimental observations in Figure 5a.

As noted in the discussion of Figure 5b, aging qualitatively changes everything about the second harmonic NLEIS spectrum; that behavior is mirrored by our model. As expected, symmetric charge transfer coefficients $\left(\alpha_{a}=\alpha_{c}=0.5\right)$, for both electrodes, produce a low frequency curve that goes straight toward the origin, as seen with the "fresh cell" model parameters. In this case, there are no mid-frequency kinetic/capacitive features. Introducing asymmetry in the transfer coefficients of the positive electrode, $\left(\alpha_{a, p o s}=0.55, \alpha_{c, p o s}=0.45\right)$ results in the characteristic second harmonic kinetics arc seen in Figures $5 \mathrm{~b}$ and $6 \mathrm{~b}$. At low frequencies, the changes in thermodynamic and diffusion coefficients for both electrodes drive a radically different low frequency second harmonic NLEIS response, despite having only modest impact on the linear EIS response. The impact of charge transfer symmetry on the second harmonic signature is further illuminated in Figure 7. The effect of varying the positive electrode anodic transfer coefficient, $\alpha_{a, p o s}$, for three cases where 


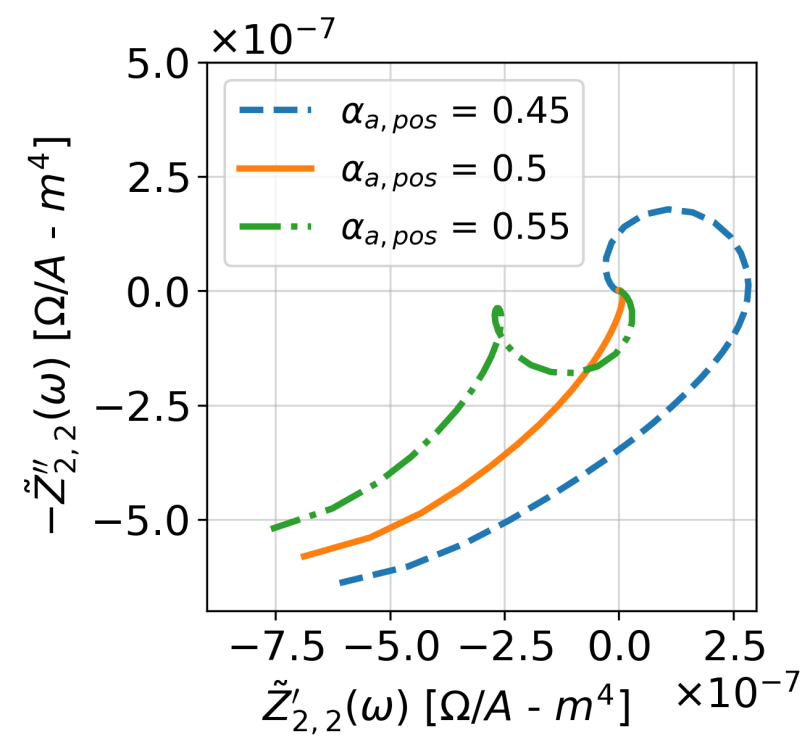

Figure 7: Nyquist plots of simulated second harmonic spectra, $\tilde{Z}_{2,2}\left(\omega_{1}\right)$ for varying positive electrode anodic transfer coefficients, $\alpha_{a, p o s}\left(\right.$ with $\left.\alpha_{c, p o s}=1-\alpha_{a, p o s}\right)$.

$\alpha_{a, p o s}=\alpha_{c, p o s}, \alpha_{a, p o s}>\alpha_{c, p o s}$ and $\alpha_{a, p o s}<\alpha_{c, p o s}$, is shown. The result shows that, depending on the nature of the asymmetry, the "second harmonic charge transfer resistance" can be positive or negative and its sign defines the quadrant of the resulting kinetic/capacitive arc. A similar, but inverted, dependence on symmetry exists for the negative electrode, because of the effect Equation 9 has on the total cell response. We are exploring scaling relationships and analytical approaches for relating the second term of Equation 11 to the quantity we refer to as the "second harmonic charge transfer resistance", which we think of as a nonlinear analog to the Randles' circuit; we will report on those results later.

\subsection{Conclusions and Implications}

Nonlinear electrochemical impedance spectroscopy (NLEIS) can be implemented as an add-on to traditional linear EIS by using a modestly larger input current or voltage modulation and advanced signal processing. Our experiments and analysis show that NLEIS provides complementary information to EIS; this new information helps unlock insights into the charge-transfer kinetics, thermodynamics, and mass transport processes that govern lithium-ion batteries. We lay out the experimental method and complications, as well as the mathematical framework for analysis of NLEIS. State-ofcharge and state-of-health data for a fresh and cycled commercial Samsung LiNMClC cell is used to illustrate the sensitivity of NLEIS.

There is a general notion that the existence of higher harmonics in the output voltage or current spectrum necessarily means EIS data is corrupted by nonlinear phenomenon. ${ }^{14-18}$ Instead, we show that the general mathematical form of the weakly nonlinear regime does not support this as a universal premise. Specifically, assuming current is modulated with amplitude $\Delta I$, the mathematical truncation error associated with a linear impedance measurement is $\sim O\left(\Delta I^{3}\right)$, whereas the second harmonic output is an $\sim O\left(\Delta I^{2}\right)$ signal. Thus, it is generally possible to find input modulation amplitudes where first order (EIS) and second order (second harmonic NLEIS) effects dominate third order effects (error in the EIS signal and third harmonic NLEIS). Of course, the exception to this statement is a symmetric electrochemical system with identical electrodes; the second harmonic 
output is identically zero at all frequencies for a symmetric cell. Fortunately, functional whole batteries are always asymmetric cells. For our experiments with $1500 \mathrm{mAh}$ power cells, input current modulations up to $500 \mathrm{~mA}$ produced fundamental peaks on the order of $10 \mathrm{~s}$ of millivolts, second harmonics several orders of magnitude smaller, and no measurable third harmonic. Experiments showed that EIS results acquired under these conditions matched much smaller modulations, and satisfied Kramers-Kronig relationships to within $1 \%$ error at all frequencies.

It is worth reiterating here that instrumental limitations constrained the usable frequency range for second harmonic NLEIS spectra to $\omega_{1} \leq 10 \mathrm{~Hz}$ because of the total harmonic distortion that was generated by the galvantostat for these low impedance cells. Thus, we were unable to achieve high enough frequencies to fully probe the negative electrode kinetic/capacitive regime. It is worth noting, however, if the higher frequency electrode possessed asymmetric charge transfer, the effect would be reflected in the lower frequency second harmonic as a real axis offset from the origin. Consequently, improvements in instruments that enable larger current modulations for low impedance batteries are crucial for accurately probing smaller, and higher frequency, physicochemical processes with NLEIS.

A tantalizing view into the power of combining NLEIS experiments with NLEIS physicsbased models was provided in the analysis of cell aging. We used a previously developed physicsbased linear EIS and second harmonic NLEIS model to interpret the second harmonic responses sensitivity to charge transfer symmetry at mid-frequency ranges, as well as thermodynamic and mass transport parameters at low frequencies. The aged batteries, despite losing less than $1 \%$ of capacity from cycling, showed a fundamentally different signature in the nonlinear response due to the presence of asymmetric charge transfer kinetics. The measurable effects of the transfer coefficients, which have not been detectable in standard EIS, can now be monitored with aging, and at various states of the battery. This ability to more completely measure fundamental aspects of charge transfer, such as symmetry coefficients in situ and in whole cells, is a potentially important advance. We are working on the tools to extract quantitative parameters from combined EIS and second harmonic NLEIS.

\section{Acknowledgments}

This work has been partially supported by funding from the Boeing-Sutter Endowment, as well as an U.S. NSF Integrative Graduate Education and Research Training grant fellowship to M.D.M (DGE-1258485) and NSF National Research Training Grant fellowship to V.W.H (DGE-1633216). This work was performed in the advanced characterization labs at the Washington Clean Energy Testbeds, an open access instrumentation facility managed by the University of Washington Clean Energy Institute.

\subsection{Appendix}

Table A.1: Voltages for NLEIS measurements

\begin{tabular}{rccccc} 
State-of-Charge & $10 \%$ & $30 \%$ & $40 \%$ & $50 \%$ & $60 \%$ \\
\hline Voltage & 3.500 & 3.643 & 3.706 & 3.820 & 3.920 \\
\hline
\end{tabular}


Table A.2: Parameters for simulating fresh and aged NLEIS spectra

Physicochemical Parameters

\begin{tabular}{|c|c|c|c|c|c|c|c|}
\hline Name & Units & $\begin{array}{c}\text { Fresh } \\
\text { Cell }\end{array}$ & $\begin{array}{c}\text { Aged } \\
\text { Cell }\end{array}$ & Name & Units & $\begin{array}{c}\text { Fresh } \\
\text { Cell }\end{array}$ & $\begin{array}{l}\text { Aged } \\
\text { Cell }\end{array}$ \\
\hline Brugg & - & 4 & 4 & $l_{n e g}$ & $\mu m$ & 88 & 88 \\
\hline$C_{d l, n e g}$ & $\mu F / \mathrm{cm}^{2}$ & 10 & 50 & $l_{\text {sep }}$ & $\mu m$ & 25 & 25 \\
\hline$C_{d l, p o s}$ & $\mu F / \mathrm{cm}^{2}$ & 25 & 1000 & $l_{\text {pos }}$ & $\mu m$ & 80 & 80 \\
\hline$c_{0}$ & $\mathrm{~mol} / \mathrm{m}^{3}$ & 1000 & 1000 & $R_{p, n e g}$ & $\mu m$ & 2 & 2 \\
\hline$D$ & $m^{2} / s$ & $1.5 \times 10^{-10}$ & $7.5 \times 10^{-10}$ & $R_{p, p o s}$ & $\mu m$ & 2 & 2 \\
\hline$D_{s, n e g}$ & $m^{2} / s$ & $3.9 \times 10^{-14}$ & $9.8 \times 10^{-15}$ & $\epsilon_{f, n e g}$ & - & 0.0326 & 0.0326 \\
\hline$D_{s, p o s}$ & $m^{2} / s$ & $1 \times 10^{-14}$ & $2.5 \times 10^{-15}$ & $\epsilon_{f, p o s}$ & - & 0.025 & 0.025 \\
\hline$\frac{\partial U_{n e g}}{\partial c^{s}}$ & $V \cdot \mathrm{cm}^{3} / \mathrm{mol}$ & 0 & -3.21 & $\epsilon_{n e g}$ & - & 0.485 & 0.485 \\
\hline$\frac{\partial U_{p o s}}{\partial c^{s}}$ & $V \cdot \mathrm{cm}^{3} / \mathrm{mol}$ & -10 & -11.7 & $\epsilon_{s e p}$ & - & 0.724 & 0.724 \\
\hline$\frac{\partial^{2} U_{n e g}}{\partial c^{s 2}}$ & $V \cdot \mathrm{cm}^{6} / \mathrm{mol}^{2}$ & -100 & -400 & $\epsilon_{\text {pos }}$ & 一 & 0.385 & 0.385 \\
\hline$\frac{\partial^{2} U_{p o s}}{\partial c^{s}}$ & $V \cdot \mathrm{cm}^{6} / \mathrm{mol}^{2}$ & 0 & -600 & & & & \\
\hline$i_{0, n e g}$ & $A / m^{2}$ & 29.7 & 14.85 & & & & \\
\hline$i_{0, \text { pos }}$ & $A / m^{2}$ & 33.0 & 5.51 & & & & \\
\hline$t_{+}^{0}$ & - & 0.364 & 0.364 & & & & \\
\hline$\alpha_{a, n e g}$ & - & 0.5 & 0.5 & & & & \\
\hline$\alpha_{a, p o s}$ & 一 & 0.5 & 0.55 & & & & \\
\hline$\alpha_{c, n e g}$ & - & 0.5 & 0.5 & & & & \\
\hline$\alpha_{c, p o s}$ & - & 0.5 & 0.45 & & & & \\
\hline$\kappa$ & $S / m$ & 0.205 & .205 & & & & \\
\hline$\sigma_{n e g}$ & $S / m$ & 100 & 100 & & & & \\
\hline$\sigma_{p o s}$ & $S / m$ & 100 & 100 & & & & \\
\hline
\end{tabular}

\section{References}

[1] Michel Armand and J.-M. Tarascon. Building better batteries. Nature, 451(7179):652-657, 2008. DOI: $10.1038 / 451652 a$.

[2] Mark E. Orazem and Bernard Tribollet. Electrochemical Impedance Spectroscopy. The Electrochemical Society series. Wiley, Hoboken, N.J, 2008. ISBN 978-0-470-04140-6.

[3] Evgenij Barsoukov, Jong Hyun Kim, Jong Hun Kim, Chul Oh Yoon, and Hosull Lee. Effect of Low-Temperature Conditions on Passive Layer Growth on Li Intercalation Materials In Situ Impedance Study. Journal of The Electrochemical Society, 145(8):2711-2717, 1998. DOI: $10.1149 / 1.1838703$.

[4] S. S. Zhang, K. Xu, and T. R. Jow. EIS study on the formation of solid electrolyte interface in Li-ion battery. Electrochimica Acta, 51(8):1636-1640, January 2006. ISSN 0013-4686. DOI: 10.1016/j.electacta.2005.02.137.

[5] M. D. Levi and D. Aurbach. Diffusion coefficients of lithium ions during intercalation into graphite derived from the simultaneous measurements and modeling of electrochemical impedance and potentiostatic intermittent titration characteristics of thin graphite electrodes. The Journal of Physical Chemistry B, 101(23):4641-4647, 1997. DOI: 10.1021/jp9701911.

[6] Ping Yu, Branko N. Popov, James A. Ritter, and Ralph E. White. Determination of the lithium ion diffusion coefficient in graphite. Journal of The Electrochemical Society, 146(1):8-14, 1999. DOI: 10.1149/1.1391556. 
[7] Uwe Tröltzsch, Olfa Kanoun, and Hans-Rolf Tränkler. Characterizing aging effects of lithium ion batteries by impedance spectroscopy. Electrochimica Acta, 51(8-9):1664-1672, January 2006. ISSN 00134686. DOI: 10.1016/j.electacta.2005.02.148.

[8] James A. Gilbert, Javier Bareño, Timothy Spila, Stephen E. Trask, Dean J. Miller, Bryant J. Polzin, Andrew N. Jansen, and Daniel P. Abraham. Cycling Behavior of NCM523/Graphite Lithium-Ion Cells in the 3-4.4 V Range: Diagnostic Studies of Full Cells and Harvested Electrodes. Journal of The Electrochemical Society, 164(1):A6054-A6065, January 2017. ISSN 0013-4651, 1945-7111. DOI: 10.1149/2.0081701jes.

[9] Tetsuya Osaka, Daikichi Mukoyama, and Hiroki Nara. Review—Development of Diagnostic Process for Commercially Available Batteries, Especially Lithium Ion Battery, by Electrochemical Impedance Spectroscopy. Journal of The Electrochemical Society, 162(14):A2529-A2537, 2015. DOI: $10.1149 / 2.0141514$ jes.

[10] Matthew D. Murbach and Daniel T. Schwartz. Analysis of Li-ion battery electrochemical impedance spectroscopy data: An easy-to-implement approach for physics-based parameter estimation using an open-source tool. Journal of The Electrochemical Society, 165(2):A297A304, 2018. DOI: 10.1149/2.1021802jes.

[11] Marc Doyle, Jeremy P. Meyers, and John Newman. Computer simulations of the impedance response of lithium rechargeable batteries. Journal of The Electrochemical Society, 147(1): 99-110, 2000. DOI: 10.1149/1.1393162.

[12] J.R. Wilson, D.T. Schwartz, and S.B. Adler. Nonlinear electrochemical impedance spectroscopy for solid oxide fuel cell cathode materials. Electrochimica Acta, 51(8-9):1389-1402, January 2006. ISSN 00134686. DOI: 10.1016/j.electacta.2005.02.109.

[13] Stephen Fletcher. Tables of Degenerate Electrical Networks for Use in the Equivalent-Circuit Analysis of Electrochemical Systems. Journal of The Electrochemical Society, 141(7):18231826, 1994. DOI: $10.1149 / 1.2055011$.

[14] K. Darowicki. Linearization in impedance measurements. Electrochimica Acta, 42(12):17811788, 1997. ISSN 0013-4686. DOI: 10.1016/S0013-4686(96)00377-5.

[15] M. Kiel, O. Bohlen, and D.U. Sauer. Harmonic analysis for identification of nonlinearities in impedance spectroscopy. Electrochimica Acta, 53(25):7367-7374, October 2008. ISSN 00134686. DOI: 10.1016/j.electacta.2008.01.089.

[16] Bryan Hirschorn, Bernard Tribollet, and Mark E. Orazem. On Selection of the Perturbation Amplitude Required to Avoid Nonlinear Effects in Impedance Measurements. Is rael Journal of Chemistry, 48(3-4):133-142, December 2008. ISSN 00212148, 18695868. DOI: 10.1560/IJC.48.3-4.133.

[17] J.J. Giner-Sanz, E.M. Ortega, and V. Pérez-Herranz. Total harmonic distortion based method for linearity assessment in electrochemical systems in the context of EIS. Electrochimica Acta, 186:598-612, December 2015. ISSN 00134686. DOI: 10.1016/j.electacta.2015.10.152.

[18] J. J. Giner-Sanz, E. M. Ortega, and V. Pérez-Herranz. Harmonic Analysis Based Method for Perturbation Amplitude Optimization for EIS Measurements. Journal of The Electrochemical Society, 164(13):H918-H924, 2017. DOI: 10.1149/2.1451713jes.

[19] Kazimierz Darowicki. Corrosion rate measurements by non-linear electrochemical impedance spectroscopy. Corrosion Science, 37(6):913-925, 1995. DOI: 10.1016/0010-938X(95)000044. 
[20] Rik-Wouter Bosch and W. F. Bogaerts. Instantaneous corrosion rate measurement with small-amplitude potential intermodulation techniques. Corrosion, 52(3):204-212, 1996. DOI: $10.5006 / 1.3292115$.

[21] R. W. Bosch, Juliaan Hubrecht, W. F. Bogaerts, and B. C. Syrett. Electrochemical frequency modulation: A new electrochemical technique for online corrosion monitoring. Corrosion, 57 (1):60-70, 2001. DOI: 10.5006/1.3290331.

[22] Thomas Kadyk, Richard Hanke-Rauschenbach, and Kai Sundmacher. Nonlinear frequency response analysis of dehydration phenomena in polymer electrolyte membrane fuel cells. International Journal of Hydrogen Energy, 37(9):7689-7701, May 2012. ISSN 03603199. DOI: 10.1016/j.ijhydene.2012.01.148.

[23] Qing Mao and Ulrike Krewer. Sensing methanol concentration in direct methanol fuel cell with total harmonic distortion: Theory and application. Electrochimica Acta, 68:60-68, April 2012. ISSN 0013-4686. DOI: 10.1016/j.electacta.2012.02.018.

[24] D. T. Schwartz, P. Stroeve, and B. G. Higgins. Fourier Transform Methods in Hydrodynamic Modulation Voltammetry. Journal of The Electrochemical Society, 136(6):1755-1764, June 1989. DOI: $10.1149 / 1.2097006$.

[25] J. Antonio Medina and Daniel T. Schwartz. Nonlinear dynamics of modulated flow between a porous injector and an impermeable substrate. Physics of Fluids (1994-present), 8(11):28952905, 1996. DOI: 10.1063/1.869129.

[26] J. Antonio Medina and Daniel T. Schwartz. Nonlinear Dynamics of Limiting Current in the Flow-Modulated Uniform-Injection Cell. Journal of the Electrochemical Society, 144(1):155164, 1997. DOI: 10.1149/1.1837378.

[27] Satoshi Nakata, Kenichi Yoshikawa, and Takehisa Matsuda. Voltage-dependent capacitance as a probe for albumin adsorption onto a solid surface. Biophysical Chemistry, 42(2):213-220, February 1992. ISSN 03014622. DOI: 10.1016/0301-4622(92)85011-R.

[28] Satoshi Nakata, Rie Takitani, and Yoko Hirata. Discrimination of glucose from its interferences using an amperometric sensor based on electrochemical nonlinearity. Analytical Chemistry, 70 (20):4304-4308, 1998. DOI: 10.1021/ac980442h.

[29] Matthew F. Smiechowski, Vadim F. Lvovich, Sowmya Srikanthan, and Roy L. Silverstein. Non-linear impedance characterization of blood cells-derived microparticle biomarkers suspensions. Electrochimica Acta, 56(23):7763-7771, September 2011. ISSN 0013-4686. DOI: 10.1016/j.electacta.2011.03.116.

[30] Nina Harting, Nicolas Wolff, Fridolin Röder, and Ulrike Krewer. Nonlinear Frequency Response Analysis (NFRA) of Lithium-Ion Batteries. Electrochimica Acta, 248:133-139, September 2017. ISSN 0013-4686. DOI: 10.1016/j.electacta.2017.04.037.

[31] Matthew D. Murbach and Daniel T. Schwartz. Extending Newman's Pseudo-TwoDimensional Lithium-Ion Battery Impedance Simulation Approach to Include the Nonlinear Harmonic Response. Journal of The Electrochemical Society, 164(11):E3311-E3320, 2017. DOI: $10.1149 / 2.0301711$ jes.

[32] Vadim F. Lvovich and Matthew F. Smiechowski. Non-linear impedance analysis of industrial lubricants. Electrochimica Acta, 53(25):7375-7385, October 2008. ISSN 0013-4686. DOI: $10.1016 /$ j.electacta.2007.12.014. 
[33] Ning $\mathrm{Xu}$ and D. Jason Riley. Nonlinear analysis of a classical system: The Faradaic process. Electrochimica Acta, 94:206-213, April $2013 . \quad$ ISSN 00134686. DOI: 10.1016/j.electacta.2013.01.141.

[34] C. Heubner, M. Schneider, and A. Michaelis. Investigation of charge transfer kinetics of Li-Intercalation in LiFePO4. Journal of Power Sources, 288:115-120, August 2015. ISSN 03787753. DOI: 10.1016/j.jpowsour.2015.04.103.

[35] Nina Harting, René Schenkendorf, Nicolas Wolff, and Ulrike Krewer. State-of-Health Identification of Lithium-Ion Batteries Based on Nonlinear Frequency Response Analysis: First Steps with Machine Learning. Applied Sciences, 8(5):821, May 2018. DOI: 10.3390/app8050821.

[36] Nina Harting, Nicolas Wolff, and Ulrike Krewer. Identification of Lithium Plating in LithiumIon Batteries using Nonlinear Frequency Response Analysis (NFRA). Electrochimica Acta, 281:378-385, August 2018. ISSN 0013-4686. DOI: 10.1016/j.electacta.2018.05.139.

[37] Nicolas Wolff, Nina Harting, Marco Heinrich, Fridolin Röder, and Ulrike Krewer. Nonlinear Frequency Response Analysis on Lithium-Ion Batteries: A Model-Based Assessment. Electrochimica Acta, 260:614-622, January 2018. ISSN 0013-4686. DOI: 10.1016/j.electacta.2017.12.097.

[38] Anup Barai, Gael H. Chouchelamane, Yue Guo, Andrew McGordon, and Paul Jennings. A study on the impact of lithium-ion cell relaxation on electrochemical impedance spectroscopy. Journal of Power Sources, 280:74-80, April 2015. ISSN 03787753. DOI: 10.1016/j.jpowsour.2015.01.097.

[39] Matthew D. Murbach, Victor W. Hu, and Daniel T. Schwartz. Data and Supplementary Notebook for "Lithium-Ion Battery Characterization with Nonlinear Electrochemical Impedance Spectroscopy: Experimental approach, insights, and initial findings". 2018. DOI: https://osf.io/tdnwa/.

[40] M. Schönleber, D. Klotz, and E. Ivers-Tiffée. A Method for Improving the Robustness of linear Kramers-Kronig Validity Tests. Electrochimica Acta, 131:20-27, June 2014. ISSN 0013-4686. DOI: 10.1016/j.electacta.2014.01.034.

[41] Salim Erol and Mark E. Orazem. The influence of anomalous diffusion on the impedance response of LiCoO2/C batteries. Journal of Power Sources, 293:57-64, October 2015. ISSN 03787753. DOI: 10.1016/j.jpowsour.2015.05.047.

[42] Jean-Marcel Atebamba, Joze Moskon, Stane Pejovnik, and Miran Gaberscek. On the Interpretation of Measured Impedance Spectra of Insertion Cathodes for Lithium-Ion Batteries. Journal of The Electrochemical Society, 157(11):A1218, 2010. ISSN 00134651. DOI: $10.1149 / 1.3489353$.

[43] B. Saha, K. Goebel, S. Poll, and J. Christophersen. Prognostics Methods for Battery Health Monitoring Using a Bayesian Framework. IEEE Transactions on Instrumentation and Measurement, 58(2):291-296, February 2009. ISSN 0018-9456, 1557-9662. DOI: 10.1109/TIM.2008.2005965.

[44] Manan Pathak, Matthew D. Murbach, Chintan Pathak, Tae-Jin Jang, Yanbo Qi, Daniel T. Schwartz, and Venkat R. Subramanian. Fast Impedance Simulation of Lithium-Ion Batteries with Pseudo-Two Dimensional Electrochemical Models. Journal of The Electrochemical Society, 165(7):A1324-A1337, January 2018. ISSN 0013-4651, 1945-7111. DOI: $10.1149 / 2.0831805$ jes. 